PPPL- -2569

DE89 004630

\title{
GYROKINETIC ENERGY CONSERVATION AND POISSON-BRACKET FORMULATION
}

\author{
A. BRIZARD \\ Plasma Physics Laboratory, Princeton University \\ Princeton, New Jersey 08543
}

\begin{abstract}
An integral expression for the gyrokinetic total energy of a magnetized plasma with general magnetic field configuration perturbed by fully electromagnetic fields was recently derived through the use of a gyro-center Lie transformation. We show that the gyrokinetic energy is conserved by the gyrokinetic Hamiltonian flow to all orders in perturbed fields. This paper is concerned with the explicit demonstration that a gyrokinetic Hamiltonian containing quadratic nonlinearities preserves the gyrokinetic energy up to third order. The Poisson-bracket formulation greatly facilitates this demonstration with the help of the Jacobi identity and other properties of the Poisson brackets.
\end{abstract}

\section{DISCLAIMER}

\begin{abstract}
This report was prepared as an account of work sponsored by an agency of the United Stoles Government. Neither the United States Governmedt nor any egency thereol, nor any of their employees, makes any warranty, express of implied, of assumes any legal liability or responsibility for the accuracy. completeness, or usefuiness of any information, apparalus, product, or process disclosed, or represents that its use would not infringe privately owned rights. Refertrise herein to any specific commercial produet, process, ar service by trade name, trodemort manufaclurer, of otherwise does not nocessarily constilute or imply its endorsement, recon. merdation, or favoring by the United States Goverament er any agency thercof. The views and opinions of authors expressed berein do not necessarily state or reflect these of the Unitad States Government or any agency thereof.
\end{abstract}




\section{i. Introduction}

Conservation laws provide us with powerful tools in the study of nonlinear dynamical systems, as the symmetry characteristics of the former allow reduction (in the sense of dynamical variables) of the latter. In the case of a Hamiltonian system, the reduced dy-namics are generated by a reduced Hamiltonian vector field. ${ }^{1.2}$ It is important, however, that the original conservation laws be also preserved in their reduced form. i.e., that the reduced Hamiltonian flow satisfies the reduced version of the original conservation law.

Approximate conservation laws are also of interest. Indeed, because of their associated asymptotic scale separation, they allow the asymptotic expression of reduced dynamics. The pioneering work of Littlejohn ${ }^{3-5}$ on the application of Lie transformation methods to the development of a Hamiltonian guiding-center theory of charged particle motion has provided much of the impetus for the establishment of Hamiltonian gyrokinetics. Within Hamiltonian guiding-center theory, Littlejohn demonstrated both energy and (angular) momentum conservation to all orders in single particle moion, ${ }^{5}$ and elegantly rederived expressions for the first and second adiabatic invariants. ${ }^{3-6}$

In Hamiltonian gyrokinetics, the unperturbed guiding-center Hamiltonian system is perturbed by electromagnetic fields with spatial and time scales that permit asymptotic analysis. ${ }^{\top}$ The asymptotic reduction is carried through by a transformation to gyro-center coordinates, characterized by the adiabatic invariance of the gyrokinetic magnetic moment, canonically conjugate to the gyro-angle. The reduced dynamics are, therefore, expressed in terms of a gyrokinetic Hamiltonian with no gyro-angle dependence. ${ }^{8-15}$

The issue of the preservation of the gyrokinetic total energy by the gyrokinetic Hamiltonian flow was discussed by various authors, but due to the tediousness of the calculations iwvolved. mixed results have been obtained. ${ }^{9,13,14,18}$ By using the algebraic simplicity associated with the Poisson-bracket formulation, we express our previous gyrokinetic treatment ${ }^{15}$ in terms of Poisson brackets, and show, conclusively, that the total gyrokinetic energy is conserved by the gyrokinetic Hamiltonian flow, to all orders in our asymptotic analysis.

This result is, in fact, not surprising at all, due to the Hamiltonia: atature of the method used. $^{3-5}$ The necessity, however, to keep all relevant nonlinear terms in our gyrokinetic Maxwell-Vlasov system, ${ }^{15}$ makes the explicit demonstration of this result valuable and represents an important test on the correctness of our nonlinear gyrokinetic system.

\section{Poisson-bracket Formulation of Gyrokinetics}

1. Gyro-center Transformation. The gyro-center Lie transformation intraduced in Ref. 15, using the action-variational Lie perturbation method of Littlejohn ${ }^{16}$ and Cary and Littlejohn, ${ }^{17}$ was devised so that the gyrokinetic Hamiltonian (and, consequently, the equations of motion) would not depend on the gyro-angle. The generating vector fields 
cartying the transformation to gyro-center coordinates $Z^{a}=\left(X_{1}, \rho_{l}, \mu . \zeta, w, t\right)$. using the compact Poisson-bracket formulation, are given as

$$
G_{10}^{\gamma}=\delta \mathbf{A}_{0} \cdot\left\{\mathbf{X}-\rho_{0}, Z^{\circ}\right\}_{0}-\left\{S_{10}, Z^{\circ}\right\}_{0},
$$

for the first-order generating vector field. and

$$
G_{20}^{\alpha}=\left\{S_{20} . Z^{\alpha}\right\}_{0}-\frac{1}{2}\left\{S_{10=} \delta \mathbf{A}_{0}\right\}_{a} \cdot\left\{\mathbf{X}+\rho_{0} . Z^{\alpha}\right\}_{0}+\frac{1}{2}\left\{S_{10}, \mathbf{X}+\rho_{0}\right\}_{0} \cdot\left\{\delta \mathbf{A}_{n} . Z^{\alpha}\right\}_{0} .
$$

for the second-order generating vector field. The operator $\left(T_{G r 0}^{-}\right)^{-1}$, involved in the evaluation of the pull-back of an arbitrary scalar function $F_{4}{ }^{16}$ is given by the expression

$$
\left(T_{G Y_{0}}^{*}\right)^{-1} F=F-G_{10}(F)-\left(G_{20}-\frac{1}{2} G_{10}^{2}\right)(F)+\cdots .
$$

The subscript $(0)$ in the previous expressions is used to indicate that the unperturbed magnetic field is taken as a uniform field.

The gyro-angle-dependent phase-space gauge functions $S_{10}$ and $S_{20}$. in the previous expressions, are chosen so that the extended phase-space canonical pair $(w, t)$ is not affected by the gyro-center transformation. ${ }^{15}$ The uniform-field Poisson bracket of two arbitrary phase-space functions is given as ${ }^{15}$

$$
\begin{aligned}
\{F, G\}_{0}= & \left(\frac{\partial G}{\partial \mu}-B \frac{\partial G}{\partial w}\right) \frac{\partial F}{\partial \zeta}-\frac{\partial G}{\partial \zeta} \frac{\partial F}{\partial \mu} \\
& +\left(\frac{\dot{b}}{B} \times \nabla_{0} G+\frac{\dot{b}}{B} \frac{\partial G}{\partial \rho_{\|}}-\rho_{\|} \mathbf{B} \frac{\partial G}{\partial w}\right) \cdot \nabla_{0} F-\left(\frac{\dot{b}}{B} \cdot \nabla_{0} G\right) \frac{\partial F}{\partial \rho_{! !}} \\
& +\left(\frac{\partial G}{\partial t}+\rho_{\|} \mathbf{B} \cdot \nabla_{0} G+B \frac{\partial G}{\partial \zeta}\right) \frac{\partial F}{\partial w}-\frac{\partial G}{\partial w} \frac{\partial F}{\partial t}
\end{aligned}
$$

Finally, che perturbation fields $\left(\delta \chi_{0}: \delta \phi_{0}\right.$ or $\left.\delta A_{0}\right)$ have the following functional dependence: $\delta \chi_{0}=\delta \chi_{0}\left(\mathbf{X}+\rho_{0}, t\right)$, where the gyroradius is denoted $\rho_{0}$. We note that because of this particular dependence, we have $\left\{\delta_{\chi_{0}} X+\rho_{0}\right\}_{0}=0$, and $\left\{\delta \phi_{0}, \delta A_{0}\right\}_{0}=0$.

2. Gyrokinetic Hamiltonian. The gyrokinetic Hamiltonian is given by the expression (containing quadratic nonlinearities) ${ }^{15}$

$$
H=H_{0}-w+\left\langle\delta \psi_{10}\right\rangle+\left\langle\delta \psi_{20}\right\rangle+\mathcal{O}\left(\epsilon^{3}\right) .
$$

where

$$
\begin{aligned}
& \delta \dot{\psi}_{10}=\delta \phi_{0}-\mathbf{V}_{200} \cdot \delta \mathbf{A}_{0}, \\
& \delta \dot{\psi}_{20}=\frac{1}{2}\left\langle\left|\delta \mathbf{A}_{0}\right|^{2}\right\rangle-\frac{1}{2 B}\left\langle\left\{\delta \bar{\Psi}_{10}^{(1)}, \delta \dot{\psi}_{10}\right\}_{0}\right\rangle+\delta \bar{\psi}_{20},
\end{aligned}
$$


with

$$
\begin{aligned}
\delta \bar{\Psi}_{10}^{(m-1)} & =\int d \zeta \delta \tilde{\Psi}_{10}^{(m)}, \\
\delta \bar{\Psi}_{10}^{(0)} & =\delta \bar{\psi}_{10} \equiv \delta \psi_{10}-\left\langle\delta \psi_{10}\right\rangle
\end{aligned}
$$

and $\mathbf{V}_{g \mathrm{r}}$ is the unperturbed particle selocity. The angular brackets $(\ldots$, in Eq. $\quad(A)$ represent gyro-angle averaging. Finally, the expressions for $S_{10}$ and $S_{20}$ are given as

$$
\begin{aligned}
& S_{10}=\frac{1}{B} \delta \bar{\Psi}_{10}^{(1)}-\frac{1}{B^{2}}\left(\frac{\partial}{\partial t}-\rho_{\mid} \mathbf{B} \cdot \nabla_{0}\right) \delta \bar{\Psi}_{10}^{(2)}+O\left(\epsilon^{3}\right) . \\
& S_{20}=\frac{1}{B} \delta \bar{\Psi}_{20}^{(1)}-O\left(\epsilon^{3}\right) .
\end{aligned}
$$

where $\epsilon$ is the small parameter involved in the definition of the (nonlinear) gyrokinetic ordering. ${ }^{?}$

\section{Gyrokinetic Energy Conservation}

1. Energy Conservation Equation. The expression for the gyrokinetic total energy was derived in Ref. 15 and was given as

$$
E_{G 10}=\int B^{2}\left\langle\left(T_{G Y_{0}}^{*}\right)^{-1} H_{0}\right\rangle F^{1} d^{8} \mathbf{Z}+\frac{1}{8 \pi} \int\left(|\delta \mathbf{E}|^{2}+|\mathbf{B}+\delta \mathbf{B}|^{2}\right) d^{3} \mathbf{x},
$$

where $\left.\left(T_{\dot{G} Y_{0}}^{*}\right)^{-1} H_{0}\right)=H-\left(\left(T_{\dot{G} r_{0}}\right)^{-1} \delta \phi_{0}\right)$. The gyro-particle distribution function is denoted by $F$, and the perturbation electromagnetic fields satisfy the gyrokinetic version of Maxwell's equations. From this expression, the energy conservation equation was given as

$$
\frac{\delta E}{\delta t} \equiv \frac{\partial H}{\partial t}-\frac{d}{d t}\left\langle\left(T_{G Y_{0}}^{*}\right)^{-1} \delta \phi_{0}\right\rangle-\left\langle\left(T_{G Y_{0}}^{*}\right)^{-1}\left(\mathbf{V}_{\theta<0} \cdot \delta E_{0}\right)\right\rangle
$$

Viote that this equation depends entirely on the single gyro-particle dynamics and, therefore. does not involve the gyro-particle distribution function (compare with Ref. 18).

Using the expression for the gyrokinetic Hamiltonian, Eq. (4), it is easy to show that

$$
\frac{\partial H}{\partial t}=\left\langle\left(T_{G r o}^{*}\right)^{-1} \frac{\partial}{\partial t} \delta \psi_{10}\right\rangle
$$

where

$$
\frac{\partial}{\partial t} \delta \psi_{10}=\left(\frac{\partial}{\partial t} \delta \phi_{0}+\left\{\delta \phi_{0}, H_{0}\right\}_{0}\right)+\mathbf{V}_{0=0} \cdot \delta \mathbf{E}_{0}
$$

so that the energy conservation equation is, therefore, given as

$$
\frac{\delta E}{\delta t}=\left\langle\left(T_{G \gamma_{0}}^{*}\right)^{-1} \frac{d}{d t} \delta \phi_{0}\right\rangle-\frac{d}{d t}\left\langle\left(T_{G Y_{0}}^{*}\right)^{-1} \delta \phi_{0}\right\rangle .
$$


The total time derivative in the first term of the right-hand side of Eq. (9) is the unperturbed time derivative

$$
\frac{d}{d t}=\frac{\partial}{\partial t}-\rho_{i} \mathbf{B} \cdot \Gamma-B \frac{\partial}{\partial \zeta} .
$$

whereas the Lotal time derivative in the second term of the right-hand side of Eq. (9) is the perturbed (gyrokinetic) time derirative

$$
\frac{d}{d t}=\frac{\partial}{\partial t}-\rho_{\mid i} \mathbf{B} \cdot \nabla-\left\{\cdot\left\langle\delta \psi_{10}-\delta w_{20}\right\rangle\right\}_{0}
$$

The explicit expression for the energy conservation equation is given as

$$
\begin{aligned}
\frac{\delta E}{\delta t}= & \left.\left\{\delta \delta v_{10}^{\prime 0}\right\rangle,\left(\delta \phi_{0}\right)\right\}_{0}-\left\langle\left\{\delta \psi_{10}^{\prime}, \delta \phi_{0}\right\}_{0}\right\rangle \\
& \left.+i\left\{\delta \psi_{10}^{-}-\left\{S_{10},\left\langle\delta \psi_{10}\right)\right\}_{0}, \delta \phi_{0}\right\}_{0}\right\rangle+\left\langle\left\{S_{10},\left\{\delta \phi_{0},\left\langle\delta \psi_{10}\right\rangle\right\}_{0}\right\}_{0}\right\rangle \\
& +\left\{\left\langle\delta \psi_{20}\right\rangle,\left\langle\delta \phi_{00}\right\rangle\right\}_{0}-\left\langle\left(G_{20}-\frac{1}{2} G_{10}^{2} j\left(B \frac{\partial \delta \phi_{0}}{\partial \zeta}\right)\right\rangle+O\left(\epsilon^{4}\right)\right.
\end{aligned}
$$

lising the Poisson-bracket property known as the Jacobi identity

$$
\{f,\{g, h\}\}+\{g,\{h, f\}\}+\{h,\{f, g\}\}=0,
$$

tinis expression reduces to (ignoring terms of order $\epsilon^{4}$ )

$$
\frac{\delta E}{\delta t}=\left\{\left(\delta v_{20}\right\rangle,\left\langle\delta \phi_{0}\right)\right\}_{0}-\left\langle\left(G_{20}-\frac{1}{2} G_{10} i\left(B \frac{\partial \delta \phi_{0}}{\partial \zeta}\right)\right\}+\left\langle\left\{\left\{S_{10}, \delta \phi_{0}\right\}_{0+}\left\langle\delta \psi_{10}\right)\right\}_{0}\right\rangle .\right.
$$

2. Energy Conservation up to third order. We wish to show that $\mathrm{Eq}_{\mathrm{q}}$. (12) vanishes at order $\epsilon^{3}$. The second term on the right-hand side of Eq. (12) is given, explicitly, by the expression

$$
\begin{aligned}
-\left\langle\left(G_{20}-\frac{1}{2} G_{10}^{2}\right)\left(B \frac{\partial \delta \phi_{0}}{\partial \zeta}\right)\right\rangle= & \left\{\left\{\delta \bar{\psi}_{20}, \delta \phi_{0}\right\}_{0}\right)+\left\langle\left\{\delta \bar{\Psi}_{10}^{(1)}, \delta \mathbf{A}_{0} \cdot\left\{\mathbf{X}+\rho_{0}, \frac{\partial \delta \Phi_{0}}{\partial \zeta}\right\}_{0}\right\}_{0}\right\rangle \\
& +\frac{1}{2 B}\left\{\left\{\delta \bar{\Psi}_{10}^{(1)},\left\{\delta \bar{\Psi}_{10}^{(1)}, \frac{\partial \phi_{0}}{\partial \zeta}\right\}_{0}\right\}_{0}\right\rangle \\
& -\frac{1}{2 \boldsymbol{F}}\left\langle\left\{\left\{\delta \mathbf{A}_{0} \cdot\left\{\mathbf{X}+\rho_{0}, \delta \bar{\Psi}_{10}^{(1)}\right\}_{0}, H_{0}\right\}_{0}, \delta \phi_{0}\right\}_{0}\right\rangle \\
& +\frac{1}{2}\left\langle\delta \mathbf{A}_{0} \cdot\left\{\mathbf{X}+\rho_{0},\left\{\mathbf{X}+\rho_{0}, B \frac{\partial \delta \phi_{0}}{\partial \zeta}\right\}_{0}\right\}_{0} \cdot \delta \mathbf{A}_{0}\right\rangle .
\end{aligned}
$$

It can be further simplified by using the following identities. In the second and fifth terms on the right-hand side of this expression, we use the Jacobi identity to show that

$$
\left\{\mathbf{X}+\rho_{0}, B \frac{\partial \delta \phi_{0}}{\partial \zeta}\right\}_{0}=\left\{\mathbf{K}+\rho_{0},\left\{\delta \phi_{0}, H_{0}\right\}_{0}\right\}_{0}=\left\{\delta \phi_{0}, \mathbf{V}_{g=0}\right\}_{0}
$$


so that

$$
\left\langle\left\{\delta \bar{\Psi}_{10}^{(1)}, \delta \mathbf{A}_{0} \cdot\left\{\delta \phi_{0}, V_{s=0}\right\}_{0}\right\}_{0}\right\rangle=\left\langle\left\{\delta \tilde{\Psi}_{10}^{(1)} \cdot\left\{\delta z_{10}, \delta \phi_{0}\right\}_{0}\right\}_{0}\right\rangle
$$

and

$$
\left\{\mathbf{X}-\rho_{0},\left\{\mathbf{X}-\rho_{0}, B \frac{\partial \delta \phi_{0}}{\partial \zeta}\right\}_{0}\right\}_{0}=\left\{\delta \phi_{0,}\left\{\mathbf{X}-\rho_{0} . \mathbf{V}_{g c 0}\right\}_{0}\right\}_{0}=\left\{\delta \phi_{0}, \mathbf{I}\right\}_{0}=0,
$$

Finally, using again the Jacobi identity and integrating the grro-angle by parts. the third term on the right-hand side becomes

$$
\frac{1}{2}\left\langle\left\{\delta \bar{\Psi}_{10}^{(2)} \cdot\left\{\delta \bar{\Psi}_{10}^{(1)} \cdot \frac{\partial \delta \phi_{0}}{\partial \zeta}\right\}_{0}\right\}_{0}\right\rangle=-\left\langle\left\{\delta \bar{\Psi}_{10}^{(1)},\left\{\delta \bar{\psi}_{10}^{\prime}, \delta \phi_{0}\right\}_{0}\right\}_{0}\right\rangle+\frac{1}{2}\left\{\left\{\left\{\delta \bar{\Psi}_{10}^{(1)}, \delta \bar{\psi}_{10}\right\}_{0}, \delta \phi_{0}\right\}_{0}\right\rangle .
$$

The energ: conservation equation, Eq. (12), is thus given by the expression

$$
\begin{aligned}
\frac{\delta E}{\delta t}= & \left\langle\left\{\delta \psi_{20}+\left\{S_{10},\left\langle\delta \psi_{10}\right\rangle\right\}_{0}+\frac{1}{2}\left\{S_{10}, \delta \bar{\psi}_{10}\right\}_{0}\right.\right. \\
& \left.\left.-\frac{\vdots}{2}\left\{\delta A_{+_{0}} \cdot\left\{\mathbf{X}-\rho_{0}, S_{10}\right\}_{0}, H_{0}\right\}_{0}, \delta \phi_{0}\right\}_{0}\right\rangle .
\end{aligned}
$$

Finally, using the Poisson-bracket expression for $\iota \ldots$

$$
\begin{aligned}
\delta \psi_{20}= & \left.\frac{1}{2} \cdot \delta \mathbf{A}_{0}\right\}^{2}-\frac{1}{2}\left\{S_{10}, \delta \bar{\psi}_{10}\right\}_{0}-\left\{S_{10},\left\langle\delta \psi_{10}\right\rangle\right\}_{0} \\
& -\frac{1}{2}\left\{\delta \mathbf{A}_{0} \cdot\left\{\mathbf{X}+\rho_{0}, S_{10} H_{0},\right\}_{0} .\right.
\end{aligned}
$$

where $S_{10}=\frac{1}{B} \delta \bar{\Psi}_{10}^{(1)}$ is used see Eq. ( $(\tau) \mid$, we obtain

$$
\frac{\delta E}{\delta t}=\left\langle\delta A_{0} \cdot\left\{\delta A_{0}, \delta \phi_{0}\right\}_{0}\right\rangle=0 .
$$

\section{Discussion}

Looking at the grokinetic ordering of the two terms on the right hand side of Eq. (9), we see that the first term contains all terms of order one, two and three, and the second term contains all terms of order two, three, and four. Showing that the gyrokinetic total energy is preserved at orders one, two, and three is, therefore, the best that can be achieved with a gyrokinetic Hamiltonian containing quadratic nonlinearities.

The claim that the gyrokinetic total energy is preserved to all orders of perturbation by the gyrokinetic Harziltonian flow is, consequently, madt in the following sense: the gyrokinetic dynamics generated by the Hamiltonian

$$
H=H_{0}+H_{1}+\cdots+H_{n},
$$

where $n=1.2, \ldots$, is such that

$$
\dot{\mu}=O\left(\epsilon^{n+1}\right),
$$


and

$$
\frac{\delta E}{\delta t}=O\left(t^{n+7}\right)
$$

Tite energy conservtion equation, for the case of a general magnetic field configuration. has again the same form as Eq. (9). It is easy to show that the gyrokinetic total energy is preserved at orders $\epsilon \epsilon_{B}$ and $\epsilon^{2} \epsilon_{B}$ (provided the first-order generating vector feld contains all terms of order $\epsilon_{B}$, , where $\epsilon_{B}$ is a small parameter used to describe the magnetic nonuniformity. introduced in Ref. 15.

In the work by Dubin et al., gyrokinetic total energy is claimed not to be conserved at order three. Indeed, if the expression for $S_{10}$, Eq. (7), is truncated so that only the first-order term is kept. and is substituted into Eq. (1), then, the energy conservation equation, Eq. (9), does not ranish at order three.

In the work of Hagan. ${ }^{18}$ the proof of the energy conservation property is made diffcult through the introduction of the gyro-particle distribution function (more precisely, its unperturbed and perturbed parts). It is claimed, however, that the proof, after tedious algebra. is carried through to fourth order by using a gyrokinetic Hamiltonian containing cubic nonlinearities.

In our previous work, ${ }^{15}$ we have shown that the energy conservation equation can be entirely written in terms of the single grro-particle dynamics. In addition, in this present work, we have shown that the proof that the gyrokinetic total energy is preserved by the grokinetic Hamiltonian flow. is simplifird by the use of the Poisson-bracket formulation.

\section{ACKNOWLEDGMENTS}

The author gratefully acknowledges useftal discussions with Drs. R. B. White and J. A. Krommes. The author would also like to tliank Dr. R. G. Littlejohn for pointing out the (approximate) conservation laws of Hamiltonjan guiding-center theory. This work was supported by the U.S. Department of Energy under contract DE-AC02-76-CHO-3073. 


\section{References}

'S.Mi. Omohundro, Gemmetrc Perturbation Theory in Phystcs, (World Scientific, Singapore. 1986).

${ }^{2}$ B.A. Boghosian. Ph. D. Dissertation. Lniversity of California - Berkeley. Lawpence Berke ley Laboratory Report LJL-23241 (February, 198i).

${ }^{3}$ R.G. Littlejohn. J. Math. Phys. 20. 2445 (1979).

${ }^{4}$ R.G. Littlejohn, Phys. Fluids 24. 1730 (1981).

${ }^{5}$ R.G. Littlejohn, J. Plasma Phys. 29, 111 (1983).

${ }^{6}$ R G. Littlejuhn, Phys. Scr. T2/1, 119 (1982).

'E.A. Frieman and L. Chen, Phys. Fluids 25. 502 (1982).

${ }^{8}$ W. Lee. Phys. Fluids 26, 556 (1983).

${ }^{9}$ D.H.E. Dubin, J.A. Krommes, C. Oberman, and W.W. Lee. Phys. Fluids 26, 3524 (1983).

${ }^{10}$ S-C. Yang and D-I. Choi, Phys. Lett. A 108, 25 (1985).

"'W.K. Hagan and E.A. Frieman, Phys. Fluids 23, 2641 (1985).

${ }^{12}$ W. W. Lee, J. Comp. Phys. 72. 243 (1987).

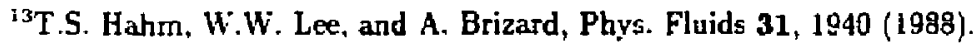

${ }^{1}$ T.S. Hahm, Phys. Flsids 31, 2670 (1988).

${ }^{15}$ A. Brizard, Princeton Plasma Physics Laboratory Report No. PPPL-2552 (September, 1988). Submitted to J. Plasma Phys.

${ }^{16}$ R.G. Littlejohn, 3. Math. Phys. 23, 752 (1982).

17J.R. Cary and R.G. Littlejohn, Ann. Phys. 151, I (1983).

${ }^{18}$ W.K. Hagan, Ph. D. Dissertation, University of Californja - San Diego, 1986. 